\title{
Detection of Spatially Correlated Objects in $3 D$ Images Using Appearance Models and Coupled Active Contours
}

\author{
Kishore Mosaliganti, Arnaud Gelas, Alexandre Gouaillard, Ramil Noche, \\ Nikolaus Obholzer, and Sean Megason \\ Department of Systems Biology, Harvard Medical School, Boston, MA - 02115, USA \\ kishore@hms.harvard.edu
}

\begin{abstract}
We consider the problem of segmenting $3 D$ images that contain a dense collection of spatially correlated objects, such as fluorescent labeled cells in tissue. Our approach involves an initial modeling phase followed by a data-fitting segmentation phase. In the first phase, cell shape (membrane bound) is modeled implicitly using a parametric distribution of correlation function estimates. The nucleus is modeled for its shape as well as image intensity distribution inspired from the physics of its image formation. In the second phase, we solve the segmentation problem using a variational level-set strategy with coupled active contours to minimize a novel energy functional. We demonstrate the utility of our approach on multispectral fluorescence microscopy images.
\end{abstract}

\section{Introduction}

Researchers in embryogenesis and cancer rely on automated segmentation of cells to understand the complex processes of tissue morphogenesis. Cell segmentation involves uniquely identifying fluorescent marked cells and organelles, such as nuclei, that are spatially correlated but whose position, number, and geometry must be determined [1]. The problem is complicated by individual variations in intensity, geometry, relative orientation and overlapping boundaries (Fig. 1).

A new aspect of the segmentation problem relates to fusing information of related structures present in multiple imaging channels. Current microscopes support high resolution imaging of as many as 32 separate channels containing information on uniquely tagged organelles. High throughput time-lapse imaging coupled with image analysis is now viewed as a tool to understand embryonic development by tracking every single cell to its ultimate fate. Therefore, segmentation tools must also be generic tracking solutions, possibly in a real-time environment coupled to the imaging.

The presence of millions of cells in dynamic imagery calls for the usage of models to drive the segmentation process. The models may be biologically inspired (packing, spatial distributions, mixing fractions, etc.), geometrical (shape, size, symmetry), or based on the physics of image formation (point spread function of the optics) [1]. Volumetric representations of objects, such as active contours, provide a natural mechanism to integrate multiple models elegantly into a segmentation exercise [2,3].

In this paper, we develop a novel fitting energy functional based on our proposed geometric and image formation models. By representing a cell as a pair (membrane + 

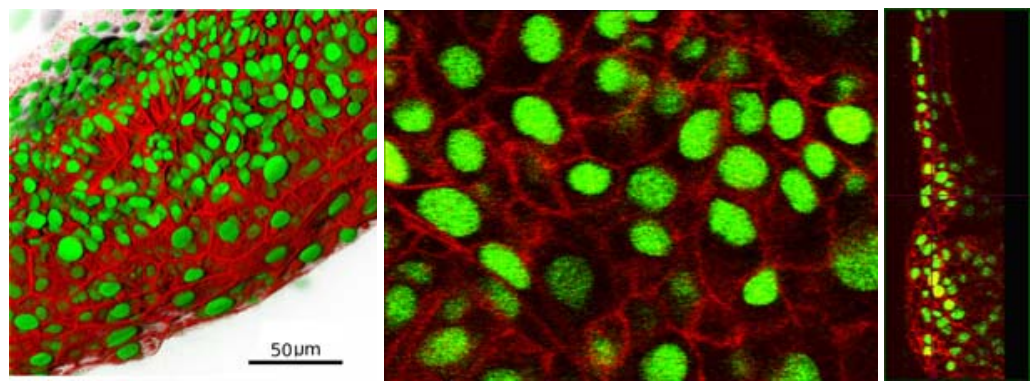

Fig. 1. Left: A $3 D$ view of the zebrafish hind-brain showing a dense collection of cells. The cell membranes are marked in red, and nuclei are in green colors. Middle: A zoomed $x-y$ image plane showing arrangement details of nuclei within membranes. Right: An $x-z$ section showing poor structural resolution of the membranes along the $z$-axis.

nucleus) of active contours, we invoke the variational level-set methodology to obtain segmentations. We also employ spatial data structures to scale our methods to large datasets of the zebrafish embryogenesis process containing millions of cells. For the rest of this paper, we describe the proposed theory simultaneously with appropriate related work for the sake of clarity.

\section{Theory and Computational Models}

For notational convenience, let $I_{m}: \Omega \rightarrow[0,255]$ and $I_{n}: \Omega \rightarrow[0,255]$ denote the observed membrane and nuclear images. We assume that there are $M$ observed cells (membrane bound with nucleus). For a cell $i$, the membrane is defined on $\psi_{i} \subset \Omega$ and the nucleus is defined on $\phi_{i} \subseteq \psi_{i}$. The background in $I_{m}$ and $I_{n}$ are defined as $\psi_{\text {out }}=\Omega-\bigcup_{i=1}^{M} \psi_{i}$ and $\phi_{\text {out }}=\Omega-\bigcup_{i=1}^{M} \phi_{i}$ respectively. Finally, let $\mathscr{N}_{\mu, \Sigma}$ denote a Gaussian distribution with mean $\mu$ and standard deviation $\Sigma$.

Brief Overview: The membrane intensity volume is sampled with 2-point correlation functions to produce a "correlation" image (Sec.2.1). This correlation image is modeled as mixture of $3 D$ spatial Gaussian functions. Additionally, the nucleus is modeled for its geometric shape as well as its intensity profile. The nucleus is given by a Gaussian shape function with constant intensity distribution within. An energy function is set up to fit the observed image data to these models, and its minimization leads to optimal settings of model parameters.

\subsection{Appearance Models}

Correlation Functions for cell shape: Membrane data is generated by tagging a fluorescent marker to point samples on cell surfaces. During imaging, the point spread function marks the membranes as thin, wispy foam structures. The data inherently has a poor SNR, creates bias fields in dense locations, and contains missing foam segments. Poor optical slicing resolution along the $z$-axis creates discontinuities in the structure 


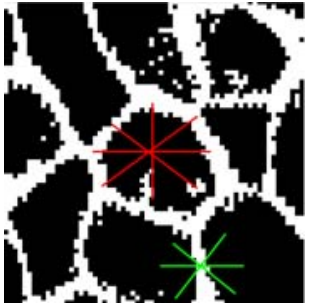

(a)

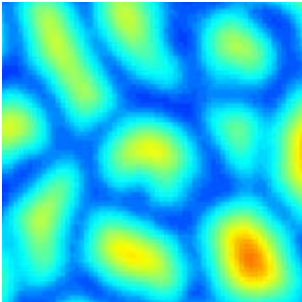

(b)

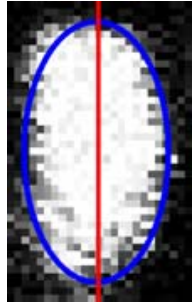

(c)

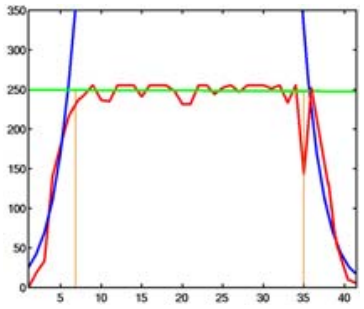

(d)

Fig. 2. (a) A thresholded image of the cell membrane showing the pcf sampling at a cell center (red) and on the periphery (green) of a cell. (b) The corresponding 2-pcf feature image is shown in the presence of binarization noise. (c) A typical nucleus with oblong shape (blue). (d) Plots of the intensity profile along a $1 D$ cross-section are shown with a constant intensity and Gaussian function fits indicated in green and blue, respectively.

thereby preventing the usage of explicit geometric models to fit intensity data. A more viable option is to implicitly model the 2-point correlation function (2-pcf) of the membrane volume [4]. Note that the membrane structure is located in the catchment basin of the 2-pcf image whose estimation is first described. Assume the presence of two materials in the image $I_{m}$, say, $O$ (background) and 1 (membranes) as shown in Fig. 2 a). Any line segment when placed in the image has its end-points located in four different configurations, namely $\{(0,0),(0,1),(1,0),(1,1)\}$. The 2-pcf $P_{i, j}^{k}: \Omega \rightarrow[0,1]$ at any point $p \in \Omega$ measures the correlation of the end-points of a line segment of given length $k$ with end-point in configuration (i,j). The following properties hold true: (i) $\sum_{i, j} P_{i, j}^{k}=1$, (ii) $P_{01}^{k}=P_{10}^{k}$, (iii) $P_{00}^{k}+P_{01}^{k}=f_{0}$ and (iv) $P_{10}^{k}+P_{11}^{k}=f_{1}$, where parameters $f_{0}$ and $f_{1}$ represent the volume fractions of the individual phases. The 2-pcfs are computationally estimated by sampling the neighborhood of a point $x \in \Omega$ with randomly oriented line segments of constant length $k$ and noting the frequencies of different configurations. We are interested in configurations where both end-points lie on the membrane, i.e. $(1,1)$. In Fig. 2(b), the pcf $P_{1,1}^{4}$ is shown as an image. The value of $k$ is chosen to be equal to the average diameter of cells $(4 \mu \mathrm{m})$ in this specific case.

Cell model: Let $C_{i}$ represent the $i^{t h}$ cell with attributes of peak intensity $a_{i}$, location $\mu_{i} \in \Omega$, and its domain $\Sigma_{i}$. Assuming that 2-pcf values fit a mixture model of Gaussian cell functions, for $p \in \psi_{i}$ we write $C_{i}(p)=a_{i} \mathscr{N}_{\mu_{i}, \Sigma_{i}}(p)$ and 0 elsewhere.

Nucleus shape and distribution: The nucleus has a convex geometric shape containing a fluorescent marker tagged to DNA proteins (Fig. 22(c)). Ideally, the marker is uniformly distributed within. During imaging, the point spread function (PSF, say $\mathscr{P}$ ) of the instrument combines light sources from neighboring voxels to produce a blurred image that is computationally equivalent to a convolution operation $I * \mathscr{P}$. The nucleus boundaries have an intensity gradient while retaining a constant intensity profile well within (Figs. 2(c)-(d)).

Nucleus model: Suppose $g($.$) is a thresholded image of the gradient magnitude image$ as given in Eq. 1. Let $N_{i}(p)$ denote the intensity profile of the $i^{\text {th }}$ nucleus given by the piecewise sum of a constant intensity region $\left(c_{i}\right)$ with a Gaussian function at boundaries. 


$$
g(p)= \begin{cases}1 & \text { if }\left|G_{\sigma} * I(p)\right|>\Gamma, \quad N_{i}(p)=g(p) a_{i} \mathscr{N}_{\mu_{i}, \Sigma_{i}}(p)+(1-g(p)) c_{i} \\ 0 & \text { else. }\end{cases}
$$

\subsection{Variational Level Sets}

Related Work: In [5], Mumford and Shah formulated the image segmentation problem as follows: given an image $I$, find a contour $S$ which segments the image into nonoverlapping regions. They proposed the following functional:

$$
F^{M S}(u, S)=\int_{\Omega}(u-I)^{2} d p+\int_{\Omega \backslash S}|\nabla u|^{2} d p+\mu|S|
$$

where $S$ is a contour that segments the original image $I$ and $u$ is a piecewise smooth approximation of $I$. The first term computes the difference in intensities between $u$ and $I$ while the second term ensures the smoothness of $u$ everywhere except on the contour $S$. The last term is a regularization for selectively obtaining smooth contours of $S$ with a user-defined weight $\mu$. In practice, it is difficult to minimize this functional since there are no bounds on shape/topology of the unknown contour $S$ of lower dimension and the non-convexity of the functional. Later, Chan and Vese [2] proposed an energy that is a piece-wise constant (PC) approximation of their functional:

$$
F^{C V}\left(S, c_{1}, c_{2}\right)=\lambda_{1} \int_{\phi_{\text {in }}}\left|I-c_{0}\right|^{2} d p+\lambda_{2} \int_{\phi_{\text {out }}}\left|I-c_{1}\right|^{2} d p+\mu|S|
$$

where $\phi_{\text {in }} / \phi_{\text {out }}$ are regions inside/outside of contour $S$, and $c_{0}$ and $c_{1}$ are two scalar constants that approximate the image intensities. The first two terms are often referred to as global binary fitting energy terms that seek to separate an image into two regions of constant image intensities. The last term is a regularization for selectively obtaining smooth contours. In an orthogonal development, Vese and Chan [6] extended their single level-set model to a multiphase model for segmenting multiple objects in images. This extension is applied in our work as well.

The piecewise smooth (PS) models such as [6] have overcome the difficulties of the PC models in the presence of smooth intensity variations. These models assume that the intensity function can be approximated by smooth functions inside and outside the contours and therefore, can correct intensity inhomogeneities [7]. These models do not capture spatial intensity distributions that characterize geometric image objects (for e.g. biological cells), which is of our interest.

Proposed active contour models: Based on our appearance models in Secs. 2.1 and 2.2, we define the following energy functions:

$$
\begin{aligned}
& \mathscr{F}_{m}\left(S, \alpha_{m}, \mu_{m}, \Sigma_{m}\right)=\lambda_{1, m} \sum_{i=1}^{M} \int_{\psi_{i}}\left|C_{i}-P_{11}^{k}\right|^{2} d p+\lambda_{2, m} \int_{\psi_{\text {out }}}|P|^{2} d p+\mu_{1, m}|S| \\
& \mathscr{F}_{n}\left(S, \alpha_{n}, \mu_{n}, \Sigma_{n}, c\right)=\lambda_{1, m} \sum_{i=1}^{M} \int_{\phi_{i}}\left|N_{i}-I_{n}\right|^{2} d p+\lambda_{2, m} \int_{\phi_{\text {out }}}\left|I_{n}\right|^{2} d p+\mu_{2, m}|S|
\end{aligned}
$$

where $\lambda$ 's, and $\mu$ 's are positive user-defined weights and $\left(\alpha_{\mathbf{m}}, \mu_{m}, \Sigma_{m}, c\right)$ are vectors of model parameters for each cell/nuclei. In both equations, the first term is a summation across all cells that measures the fit of the model to observed data inside the contour. The second term fits the observed background to 0 outside the contours. Note: The rest of the analysis will neglect $\lambda$ 's, and $\mu$ 's (=1) and consider the case when $M=1$. 


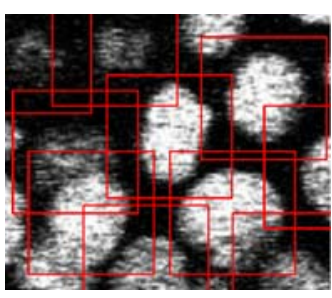

(a)
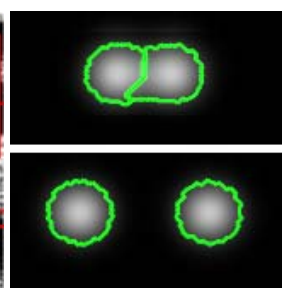

(b)

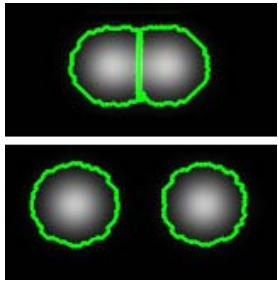

(c)

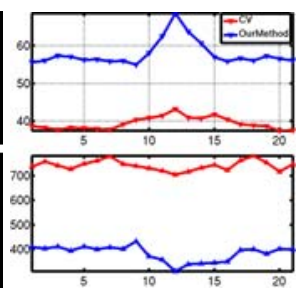

(d)

Fig. 3. (a) Rectangular regions of $2 \times$ cell diameter placed around cells to optimize calculations and limit memory usage. (b) Gaussian blob tracking using the CV method. (c) Gaussian blob tracking using the proposed method. (d) Comparisons of mean intensities (top) and observed blob sizes (bottom) across 21 time-points. Changes observed in CV method (blue) as compared to our method (red) during collision.

In level-set methods, a contour $S \subset \Omega$ is represented by the zero level-set of a Lipschitz function $\phi: \Omega \rightarrow \mathfrak{R}$. Using level-sets and Eq. 1, the above energy functionals are expanded as:

$$
\begin{aligned}
\mathscr{F}_{m}\left(\phi_{m}, \alpha_{m}, \mu_{m}, \Sigma_{m}\right) & =\int_{\Omega}\left|\alpha_{m} \mathscr{N}_{\mu_{m}, \Sigma_{m}}-P_{11}^{k}\right|^{2} H\left(\phi_{m}\right) d p+\int_{\Omega}|P|^{2} \tilde{H}\left(\phi_{m}\right) d p \\
& +\int_{\Omega} \delta\left(\phi_{m}\right)\left|\nabla \phi_{m}\right| d p+O\left(\phi_{m}, \phi_{n}\right) \\
\mathscr{F}_{n}\left(\phi_{n}, \alpha_{n}, \mu_{n}, \Sigma_{n}, c\right) & =\int_{\Omega} g\left|\alpha_{n} \mathscr{N}_{\mu_{n}, \Sigma_{n}}-I_{n}\right|^{2} H\left(\phi_{n}\right) d p+\int_{\Omega}(1-g)\left|c-I_{n}\right|^{2} H\left(\phi_{n}\right) d p \\
& +\int_{\Omega}\left|I_{n}\right|^{2} \tilde{H}\left(\phi_{n}\right) d p+\int_{\Omega} \delta\left(\phi_{n}\right)\left|\nabla \phi_{n}\right| d p+O\left(\phi_{m}, \phi_{n}\right)
\end{aligned}
$$

where $H$ is the Heaviside function, and $\tilde{H}()=.1-H($.$) . In order to encourage the$ nucleus $\left(\phi_{n}\right)$ to remain coupled within the membrane $\left(\phi_{m}\right)$, we add the overlap term $O\left(\phi_{m}, \phi_{n}\right)=v \int_{\Omega} H\left(\phi_{n}\right) \tilde{H}\left(\phi_{m}\right) d p$ to both energies. Note that when the nucleus is fully within the membrane, this term vanishes and is maximized when it does not overlap. We gain considerable synergy by fusing two separate image channels. In order to ensure the stable evolution of the level-set functions in both energy functions, we add the distance regularizing term to penalize its deviation from a signed distance function by $\mathrm{Li}$ et al. [8]. The deviation is characterized by the following integral $D(\phi)=\int_{\Omega} \frac{1}{2}(|\nabla \phi(p)|-1)^{2}$. Since Eq. 5 is a particular case of Eq. 6 when $g()=$.0 , we discuss the latter solution alone. This is a novel feature of our method in that it applies uniformly to both nuclei as well as membrane segmentation.

$$
H_{\varepsilon}(x)=\frac{1}{2}\left(1+\frac{2}{\pi} \arctan \left(\frac{x}{\varepsilon}\right)\right) \quad \delta_{\varepsilon}(x)=\frac{1}{\pi} \frac{\varepsilon}{\varepsilon^{2}+x^{2}} \quad \frac{\partial \phi}{\partial t}=-\frac{\partial \mathscr{F}}{\partial \phi}
$$

As in [2], the Heaviside and Delta functions in Eqs. 56 6] are approximated as in Eq.7] The minimization of energy is done by a Maximum Expectation procedure. For fixed parameters $(\alpha, \mu, \Sigma, c)$, we first solve the level-set evolution equation as the gradient descent equation where $\frac{\partial \mathscr{F}}{\partial \phi}$ is the the first order functional derivative of the energy 
$\mathscr{F}$. Then the minimizing parameters $(\alpha, \mu, \Sigma, c)$ are determined for a given $\phi$. Let $\alpha N_{\mu, \Sigma}(p)=e^{m \cdot u^{t}(p)}$ where $p=(x, y, z), m$ is the coefficient vector, and $u(p)$ is the trivariate monomial vector of order 2 , i.e. $u(p)=\left\{x^{i} y^{j} z^{k} ; i+j+k \leqslant 2\right\}$. The minimization of the first term in Eq. 6leads to a discrete least-squares problem:

$$
\begin{gathered}
m^{\star}=\arg \min _{m} \sum_{p \in \Omega_{i n}} g(p)\left(\ln (I(p))-m \cdot u^{t}(p)\right)^{2}=\left(M^{t} \cdot M\right)^{-1} \cdot M^{t} \cdot y \\
c=\frac{\int_{\Omega}(1-g(p)) I(p) H_{\varepsilon}(\phi(p)) d p}{\int_{\Omega}(1-g(p)) H_{\varepsilon}(\phi(p)) d p}
\end{gathered}
$$

where $M$ is a matrix of size $10 \times n$ with $M_{i j}=u_{i}\left(p_{j}\right)$ ( $n$ is the number of pixels in set $\left.\left\{p \in \Omega_{i n} \mid g(p) \neq 0\right\}\right) ; y$ is a vector of size $n$ with $y_{i}=\ln \left(I\left(p_{i}\right)\right)$. At each iteration in the level-set evolution, we solve a linear system via a Singular Value Decomposition to compute the coefficients $m^{\star}$. The constant intensity minimizer $c$ is determined as a weighted average of pixel intensities as in Eq. 9 . The multiphase case $(M>1)$ is solved as in [3] where each nucleus/cell is represented by a unique level-set function.

\section{Results and Discussion}

The proposed method for detecting deformable correlated objects in 3D multichannel intensity images was quantitatively assessed with both synthetic and real fluorescence microscopy data. In all our experiments, for the sake of objectivity, we set $\lambda_{i}=1$, $\mu_{i}=100, \Gamma=45$, and $\sigma=3$ uniformly across all cells. Our goals are three-fold:

(1) Effectiveness of models: We show superior segmentation accuracies compared to the multiphase Chan-Vese (CV) algorithm [2] that is devoid of any underlying models. This shows that our models effectively represent underlying structures. First, we track a pair of isotropic Gaussian blobs ( $\alpha=100, \Sigma=10$ pixel units) over 21 time-points as they collide (center separation of 15 pixel units at $t=11$ ) and move apart. We show two time-points when they are closest and farthest apart for the CV and our method (Figs. 3 b) and (c)). The CV algorithm segments cells until the closest point where the cell contours do not represent the object shape truly. Our method recognizes the underlying Gaussian distribution and effectively fits the parameters even during close contact. Note that the CV algorithm neglects the Gaussian tails. In Fig. 3 (c), we show plots of the mean intensity and sizes of the objects across time-points. Our algorithm (in blue) respects the underlying Gaussian distribution and shape and hence maintains a consistent size (larger) and mean (lower) across all time-points. Fig. 4 (a) shows a $2 D$ cellular microstructure constructed by modeling membranes using weighted Voronoi diagrams [9]. The nucleus model consists of randomly oriented and normally varying elliptic shapes and intensities $(150 \pm 75)$. The images $(500 \times 500 \times 100)$ are convolved with an experimentally determined PSF $(0.15 \mu \mathrm{m} \times 0.15 \mu \mathrm{m} \times 0.75 \mu \mathrm{m})$ and degraded with Poisson $\left(\mu=p_{i}\right)$ and Gaussian noise $\left(0, p_{i}\right)$ where $p_{i}$ is the pixel intensity. The CV method responds to absolute intensity values alone and spreads to neighboring cell regions leading to their shrinkage (Fig.4(b)). In Fig. 5, we tabulate the Dice metric computed on nuclei and membrane segmentations separately for five phantom images (ground-truth) with 


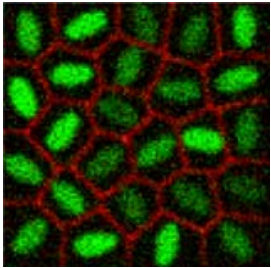

(a)

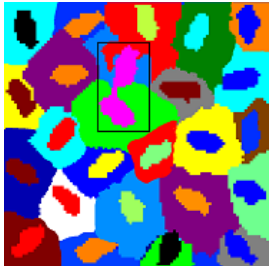

(b)

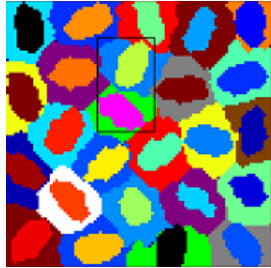

(c)

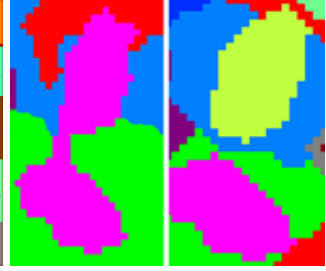

(d)

Fig. 4. (a) Phantom cell constructed from foam models, our proposed nucleus model and noise. (b) CV segmentation with enlarged cell boundaries. (c) Segmentation with the proposed method respects underlying intensity and shape distributions. (d) An instance of coupling benefits.

their with increasing noise levels. We apply $\{0.5,0.75,1,1.5,2\}$ multiplicative factors to $p_{i}$ for setting the mean and variance in our noise models. The Dice metric on two segmentations $A$ and $B$ measures the ratio of their intersection $(A \cap B)$ to their union $(A \cup B)$. The result of our method is shown in Fig. 4 ( $\mathrm{c})$.

Fig. 5 shows the output of our algorithm on zebrafish spinal cells (Fig. 2(a)). These datasets have pixel dimensions of $1024 \times 1024 \times 100$, pixel spacings of $0.2 \times 0.2 \times$ $1 \mu \mathrm{m}^{3}$, file-size of $143 \mathrm{MB}$, and contain 7896 detected cells. The algorithm took $63 \mathrm{~min}-$ utes to execute, excluding the pcf calculations for the membrane channel. We devised a novel way to validate our result using special staining protocols. While the entire membrane channel was fluorescent tagged, only a few random nuclei (48) were selectively tagged. This allowed us to segment cells using the membrane channel, and the nuclei were estimated by setting the nucleo-cytoplasmic ratio to 0.7 . In the spinal cells, cells are mostly spherical with a centric-nucleus. The estimated nuclei were compared with the scattered nuclei by an expert on the basis of correspondence (42 matches), total number of cells found, visual inspection, and the volume distributions (not shown) observed. The incorrect matches resulted from boundary cells with partial/broken membranes and over-segmented large cells.
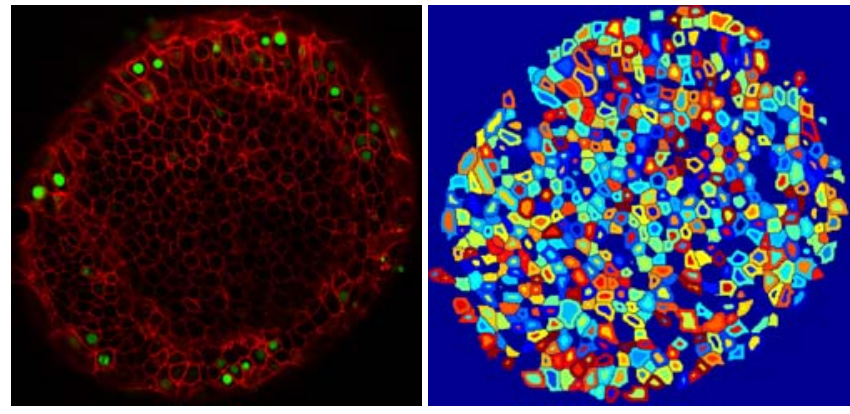

\begin{tabular}{|c|c|c|}
\hline \multirow{2}{*}{ Dataset } & \multicolumn{2}{|c|}{ Nuclei } \\
\cline { 2 - 3 } & CV & Our Method \\
\hline 1 & 0.866 & 0.9443 \\
2 & 0.8421 & 0.9252 \\
3 & 0.8132 & 0.9132 \\
4 & 0.8143 & 0.8842 \\
5 & 0.78 & 0.8632 \\
\hline \multicolumn{3}{|c|}{ Membrane } \\
\hline 1 & 0.9832 & 0.9875 \\
2 & 0.9342 & 0.9563 \\
3 & 0.9024 & 0.9346 \\
4 & 0.8623 & 0.9274 \\
5 & 0.8134 & 0.9123 \\
\hline
\end{tabular}

Fig. 5. Left: A $2 D$ optical slice of zebrafish spinal cells with membranes and a few random nuclei. Middle: Segmentation of the membranes with estimated nuclei. Right: Dice metric computed on five phantom datasets for the nuclei and membrane segmentations separately. 
(2) Memory and running time optimization for high-throughput imaging: The per iteration running time $\left(O\left(M^{2}|\Omega|\right)\right)$ is a linear function of the number of cells $(M)$, image size $(|\Omega|)$ and the number of neighbors to each cell (potentially $M$ ). Two level-sets per cell were evolved on $\Omega$ limiting it to $\sim 10-25$ cells in our real datasets. Using ROIs of twice the cell diameter (of physical size $S=4 \mu \mathrm{m}$ radius as shown in Fig. 3 (a)) and $k$ d-trees to hold ROI centroid locations leads to drastic reductions in peak memory as well as running time $(O(M|S| \log (M)))$. The method was scalable to 7896 detected cells in Fig. 5. Using the $k$ d-tree for neighbor search led to a $38 \%$ speed-up per iteration.

(3) Synergy by coupling: In multichannel data, the coupling mechanism leads to better segmentations in the presence of noise. In Fig. 4(d), we show a single zoomed instance where superior segmentations result from using the membrane as well as the nucleus.

\section{Summary}

In this work, we formulated models that are convenient and intuitive for the practitioner, namely the existence of a spatial intensity distribution correlated with shape and combined them with cell morphology. We have presented a computationally optimized framework for cell segmentation using variational active contours. A unique feature of our method is the synergistic coupling of nuclear and membrane segmentation problems into a single formulation. We successfully segment $3 D+t$ confocal data on zebrafish embryogenesis and provide comparisons with a well-known segmentation method.

\section{References}

1. Khairy, K., Reynaud, E., Stelzer, E.: Detection of deformable objects in 3D images using markov-chain monte carlo and spherical harmonics. In: Metaxas, D., Axel, L., Fichtinger, G., Székely, G. (eds.) MICCAI 2008, Part II. LNCS, vol. 5242, pp. 1075-1082. Springer, Heidelberg (2008)

2. Chan, T., Vese, L.: An active contour model without edges. In: Scale-Space Theories in Comp. Vision, pp. 141-151 (1999)

3. Dufour, A., Shinin, V., Tajbakhsh, S., Guillon-Aghion, N., Olivo-Marin, J., Zimmer, C.: Segmenting and tracking fluorescent cells in dynamic 3-D microscopy with coupled active surfaces. IEEE Trans. Image Process 14, 1396-1410 (2008)

4. Mosaliganti, K., Machiraju, R., Leone, G.: Tensor classification of $N$-point correlation function features for histology tissue segmentation. Medical Image Analysis 13(1) (2009)

5. Mumford, D., Shah, J.: Optimal approximations by piecewise smooth functions and associated variational problems. Comm. on Pure and App. Math. 42(5), 577-685 (1989)

6. Vese, L., Chan, T.: A multiphase level set framework for image segmentation using the mumford and shah model. Intl. Journal of Comp. Vision 50, 271-293 (2002)

7. Li, C., Huang, R., Ding, Z., Gatenby, C., Metaxas, D., Gore, J.: A variational level set approach to segmentation and bias correction of images with intensity inhomogeneity. In: Metaxas, D., Axel, L., Fichtinger, G., Székely, G. (eds.) MICCAI 2008, Part II. LNCS, vol. 5242, pp. 1083 1091. Springer, Heidelberg (2008)

8. Li, C., Xu, C., Gui, C., Fox, M.D.: Level set evolution without re-initialization: A new variational formulation. In: Comp. Vision and Pattern Recogn., pp. 430-436 (2005)

9. Redenbach, C.: Microstructure models for cellular materials. Computational Materials Science 44(4), 1397-1407 (2009) 